\title{
On comparison between Cooray-Rubinstein and FDTD methods for ground conductivity effect on horizontal electric field evaluation in time domain
}

\begin{abstract}
Ground conductivity parameters are effective on the horizontal electric field due to impact of lightning near the power lines. In this paper, the FDTD method and Caligaris et al. algorithm (Cooray-Rubinstein method in time domain) are applied for evaluation of horizontal electric field in the close distance from lightning channel case. Therefore, the results are compared together.Also new method by combination of dipole method by FDTD method and Caligaris algorithm is recommended.
\end{abstract}

Keyword: Ground conductivity; Lightning; The horizontal electric field 